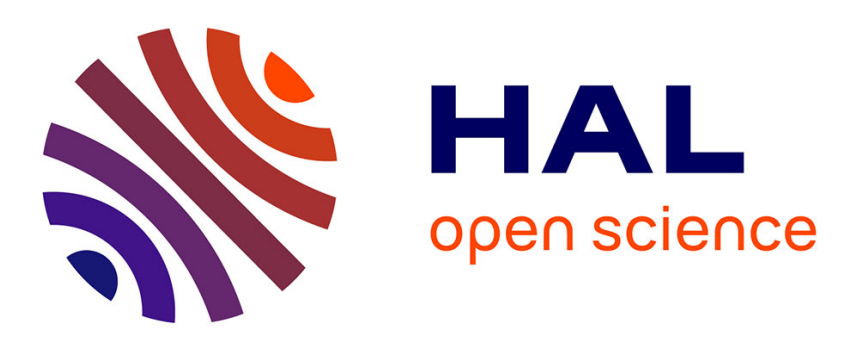

\title{
Electromechanical wave finite element method for interconnected piezoelectric waveguides
}

Boris Lossouarn, Mathieu Aucejo, Jean-François Deü

\section{To cite this version:}

Boris Lossouarn, Mathieu Aucejo, Jean-François Deü. Electromechanical wave finite element method for interconnected piezoelectric waveguides. Computers \& Structures, 2018, 199, pp.46 - 56. 10.1016/j.compstruc.2018.01.009 . hal-01739213

\section{HAL Id: hal-01739213 \\ https://hal.science/hal-01739213}

Submitted on 26 Jun 2019

HAL is a multi-disciplinary open access archive for the deposit and dissemination of scientific research documents, whether they are published or not. The documents may come from teaching and research institutions in France or abroad, or from public or private research centers.
L'archive ouverte pluridisciplinaire $\mathbf{H A L}$, est destinée au dépôt et à la diffusion de documents scientifiques de niveau recherche, publiés ou non, émanant des établissements d'enseignement et de recherche français ou étrangers, des laboratoires publics ou privés. 


\title{
Electromechanical wave finite element method for interconnected piezoelectric waveguides
}

\author{
B. Lossouarn ${ }^{\mathrm{a}, *}$, M. Aucejo ${ }^{\mathrm{a}}$, J.-F. Deü ${ }^{\mathrm{a}}$ \\ ${ }^{a}$ Structural Mechanics and Coupled Systems Laboratory, Cnam \\ 292 Rue Saint-Martin, 75003 Paris, France
}

\begin{abstract}
A novel finite element (FE) formulation involving external electrical degrees of freedom is proposed for cases dealing with the coupling of mechanical and electrical waveguides through an array of piezoelectric patches. Interconnections between successive periodic unit cells enable the use of the transfer matrix (TM) formalism. It is thus shown that the wave finite element (WFE) method can be applied to electromechanical waveguides. As both mechanical and electrical variables are enclosed in the state vectors, the resulting attenuation and phase constants define waves that propagate in the two domains. For the computation of frequency response functions, it is proposed to employ the Riccati transfer matrix (RTM) method in order to avoid numerical instability. The whole computational method is validated through two examples involving a rod and a beam coupled to passive electrical transmission lines interconnecting a piezoelectric array.
\end{abstract}

Keywords: Wave propagation, Wave finite element method, Riccati transfer matrix method, Piezoelectric coupling, Electrical network

\section{Introduction}

The idea of extending the concept of piezoelectric shunts to the synthesis of adaptive metamaterials has drawn much attention over the past decade. This actually started with distributions of independent piezoelectric patches all over a mechanical structure. Indeed, structural discontinuities induce mechanical band gaps 1, 2] that can potentially be combined with the electromechanical band gaps generated by piezoelectric shunts. Considering one-dimensional media, longitudinal wave propagation was first considered 3. 4, and the strategy involving linear resonant shunts was then applied to the control of transverse waves [5-10]. The resonant shunts were also replaced by more broadband but not passive solutions as amplified resonant shunts [11, 12, switch techniques [13, 14] and negative capacitance shunts [15, 16]. In all the aforementioned references, a periodicity of the one-dimensional structures enables the use of transfer matrix (TM) formulations to compute propagation constants as well as the global dynamics of the coupled structure [17]. The wave finite element (WFE) method [18 23] thus becomes a convenient technique when unit cells are defined from a finite element (FE) model.

A FE formulation for structures covered with piezoelectric elements was given by Thomas et al. 24] who focused on thin piezoelectric patches shunted with independent electrical circuits. The model is based on a condensation of the electrical degrees of freedom in order to recast the system into a standard mechanical formulation. However, this method is not applicable when considering interconnections of several patches through an electrical network. In this case, an electromechanical waveguide is created and a wave can propagate simultaneously in the mechanical and electrical domains. Because there are electrical nodes that interconnect successive elements, the electrical degrees of freedom cannot be condensed in the mechanical

\footnotetext{
* Corresponding author

Email address: boris.lossouarn@lecnam.net (B. Lossouarn)
} 
problem. For example, this arises when considering a structure coupled to its electrical analogue for a multimodal vibration damping purpose [25, 26] or when investigating control of wave propagation with interconnected arrays of piezoelectric elements 27 31. Based on a periodic distribution, a TM method can be implemented but it requires the use of external electrical degrees of freedom. A novel WFE formulation dedicated to electromechanical periodic waveguides is thus required.

In the next section, after providing a FE formulation for piezoelectric structures, electric charge displacement and voltage vectors are defined by analogy with displacement and force vectors. The equivalent of a dynamic stiffness matrix (DSM) is obtained from the constitutive equations of the electromechanical problem. The main difference with a purely mechanical formulation or a problem involving independent piezoelectric shunts is that the state vectors include both mechanical and electrical variables [25, 26, 29, 34]. In Section 3, the original electromechanical DSM is rearranged to bring together the left and right variables. With this partitioning, the WFE method can be applied to the coupled waveguide. A TM is thus defined after condensation of the internal mechanical degrees of freedoms. Another novelty concerns the use of the Riccati transfer matrix (RTM) method [35 38] for forced response of such a periodic electromechanical problem. This offers a convenient and numerically stable technique for the analysis of wave propagation in periodic structures involving an interconnected array of piezoelectric patches. Section 4 finally shows examples of a rod and a beam coupled to second and fourth order transmission lines, respectively. Both electrical networks are purely passive because they only involve magnetic components, as inductors and transformers, and the inherent capacitance of the piezoelectric patches. From a computation based on the proposed electromechanical TM, the propagation constants and the frequency response functions show a strong influence regarding piezoelectric coupling to an electrical network.

\section{Finite Element model}

A general FE formulation is given for elastic structures covered with thin piezoelectric patches. Then, the electromechanical matrix system is adapted to problems involving interconnections of piezoelectric patches. From the definition of electrical matrices that are equivalent to mass and stiffness matrices, energy considerations lead to an electromechanical DSM unifying both mechanical and electrical degrees of freedom.

\subsection{Finite element formulation involving piezoelectric coupling}

A convenient FE model was proposed by Thomas et al. 24] for problems involving thin piezoelectric patches connected to independent electrical circuits. The main steps are recalled in order to provide a matrix system allowing computation of the electromechanical problem. Standard indicial notations are employed in the following developments. The subscripts $i, j, k, l$ refer to the three-dimensional vectors and tensor components, repeated subscripts imply summation and comma corresponds to partial derivative. An elastic structure, occupying a domain $\Omega_{s}$, is covered with patches of piezoelectric material that represent a second domain $\Omega_{p}$. The set $\left(\Omega_{s}, \Omega_{p}\right)$ is thus a partition of the whole domain $\Omega$, which is subjected to prescribed body forces $f_{i}^{d}$. Furthermore, the domain boundary is subjected to a prescribed displacement $u_{i}^{d}$ on a part $\Gamma_{u}$ and to a prescribed surface force density $t_{i}^{d}$ on the complementary part $\Gamma_{t}$. The local equations governing the mechanical phenomena are

$$
\begin{gathered}
\sigma_{i j, j}+f_{i}^{d}=\rho \frac{\partial^{2} u_{i}}{\partial t^{2}} \quad \text { in } \Omega \\
\sigma_{i j} n_{j}=t_{i}^{d} \\
u_{i}=u_{i}^{d} \quad \text { on } \Gamma_{t}
\end{gathered},
$$

where $\sigma_{i j}$ corresponds to the linearized stress tensor, $\rho$ is the mass density, $n_{i}$ is the unit normal external to $\Omega$ and $t$ corresponds to the time. Concerning the electric boundary conditions, they are defined around the piezoelectric domain by a prescribed electric potential $\psi^{d}$ on the boundary $\Gamma_{\psi}$ and a surface density of free electric charges $q^{d}$ on the remaining part $\Gamma_{q}$. Then, based on the Gausss law with no free electric charges in the homogeneous piezoelectric material, the local equations governing the electrical phenomena are

$$
\begin{array}{cc}
D_{i, i}=0 & \text { in } \Omega_{p} \\
D_{i} n_{i}=-q^{d} & \text { on } \Gamma_{q} \\
\psi=\psi^{d} & \text { on } \Gamma_{\psi}
\end{array}
$$


where $D_{i}$ is the electric displacement vector.

The present formulation involves piezoelectric material giving rise to an electromechanical coupling described by the constitutive equations

$$
\begin{gathered}
\sigma_{i j}=c_{i j k l} \varepsilon_{k l}-e_{k i j} E_{k} \\
D_{i}=e_{i k l} \varepsilon_{k l}+\epsilon_{i k} E_{k}
\end{gathered}
$$

that relate the stress tensor and the electric displacement vector to $\varepsilon_{k l}=\frac{1}{2}\left(u_{k, l}+u_{l, k}\right)$, the linearized deformation tensor, and $E_{k}$, the electric field vector. $c_{i j k l}$ denotes the elastic moduli at constant electric field, $e_{i k l}$ the piezoelectric constants and $\epsilon_{i k}$ the dielectric permittivities at constant strain. Under the electrostatic approximation, the electric field can be described in terms of a scalar potential as

$$
E_{k}(\psi)=-\psi_{, k}
$$

This equation refers to a conservative electric field that is obtained by neglecting time variation of the magnetic field. The approximation of the Maxwell-Faraday equation is valid because the characteristic time of the electric phenomena is much smaller than the one of the mechanical phenomena. This also means that eventual electromagnetic waves are neglected, which justify the lumped approximation used for the electrical components in the following developments based on circuit theory. Another approximation relies in the fact that we only consider thin piezoelectric patches that are polarized in their transverse direction along a constant thickness $h$. The electric field vector is thus normal to the electrodes and uniform in the piezoelectric patch, so

$$
E_{k}=-\frac{V_{\mathrm{I}}}{h} n_{k},
$$

where $V_{\mathrm{I}}=\psi_{+}-\psi_{-}$is the potential difference between the electrodes that cover the upper and lower surfaces of the piezoelectric patches and $n_{k}$ is the $k^{\text {th }}$ component of the normal unit vector to the surface of the electrodes. In this paper, we consider mechanical unit cells covered with a single piezoelectric patch or a set of patches connected in parallel. This means that a single voltage $V_{\mathrm{I}}$ appears in the electromechanical formulation.

A variational formulation in terms of $\left(u_{i}, V_{\mathrm{I}}\right)$ can be deduced from the previous equations 24]. Other formulations can also be obtained, as shown for instance in Refs. 39, 40. If $\mathcal{C}_{u}$ is the space of sufficiently regular functions $u_{i}$ defined in the whole domain $\Omega$ and $\mathcal{C}_{u}^{*}=\left\{u_{i} \in \mathcal{C}_{u} \mid u_{i}=0\right.$ on $\left.\Gamma_{u}\right\}$, then

$$
\begin{aligned}
& \int_{\Omega} c_{i j k l} \varepsilon_{k l}(u) \varepsilon_{i j}(\delta u) \mathrm{d} \Omega+\frac{V_{\mathrm{I}}}{h} \int_{\Omega_{p}} e_{k i j} n_{k} \varepsilon_{i j}(\delta u) \mathrm{d} \Omega \\
& \quad+\int_{\Omega} \rho \frac{\partial^{2} u_{i}}{\partial t^{2}} \delta u_{i} \mathrm{~d} \Omega=\int_{\Omega} f_{i}^{d} \delta u_{i} \mathrm{~d} \Omega+\int_{\Gamma_{t}} t_{i}^{d} \delta u_{i} \mathrm{~d} S \quad \forall \delta u_{i} \in \mathcal{C}_{u}^{*}
\end{aligned}
$$

and

$$
-\frac{\delta V_{\mathrm{I}}}{h} \int_{\Omega_{p}} e_{i k l} \varepsilon_{k l}(u) n_{i} \mathrm{~d} \Omega+\delta V_{\mathrm{I}} C^{\varepsilon} V_{\mathrm{I}}=\delta V_{\mathrm{I}} q_{\mathrm{I}}, \quad \forall \delta V \in \mathbb{R},
$$

where $q_{\mathrm{I}}$ is the electric charge contained in the electrodes and $C^{\varepsilon}=\frac{1}{h^{2}} \int_{\Omega_{p}} \epsilon_{i k} n_{i} n_{k} \mathrm{~d} \Omega$ is the capacitance of the set of piezoelectric patches when no mechanical motion is allowed. The displacement field in the weak formulations (6) and (7) is discretized through a finite element method. Using any classical shape functions and considering the assembly procedure, the various linear and bilinear forms in the variational formulation are transformed through

$$
\begin{aligned}
& \int_{\Omega} \rho \frac{\partial^{2} u_{i}}{\partial t^{2}} \delta u_{i} \mathrm{~d} \Omega \Rightarrow \delta \boldsymbol{q}_{\boldsymbol{m}}{ }^{T} \boldsymbol{M}_{\boldsymbol{m}} \boldsymbol{q}_{\boldsymbol{m}} \\
& \int_{\Omega} c_{i j k l} \varepsilon_{k l}(u) \varepsilon_{i j}(\delta u) \mathrm{d} \Omega \Rightarrow \delta \boldsymbol{q}_{\boldsymbol{m}}{ }^{T} \boldsymbol{K}_{\boldsymbol{m}} \boldsymbol{q}_{\boldsymbol{m}} \\
& \frac{V_{\mathrm{I}}}{h} \int_{\Omega^{(p)}} e_{k i j} n_{k} \varepsilon_{i j}(\delta u) \mathrm{d} \Omega \Rightarrow \delta \boldsymbol{q}_{\boldsymbol{m}}^{T} \boldsymbol{K}_{\boldsymbol{c}} V_{\mathrm{I}} \\
& \int_{\Omega}^{h} f_{i}^{d} \delta u_{i} \mathrm{~d} \Omega+\int_{\Gamma_{t}} t_{i}^{d} \delta u_{i} \mathrm{~d} S \Rightarrow \delta \boldsymbol{q}_{\boldsymbol{m}}{ }^{T} \boldsymbol{F}_{\boldsymbol{m}}
\end{aligned}
$$




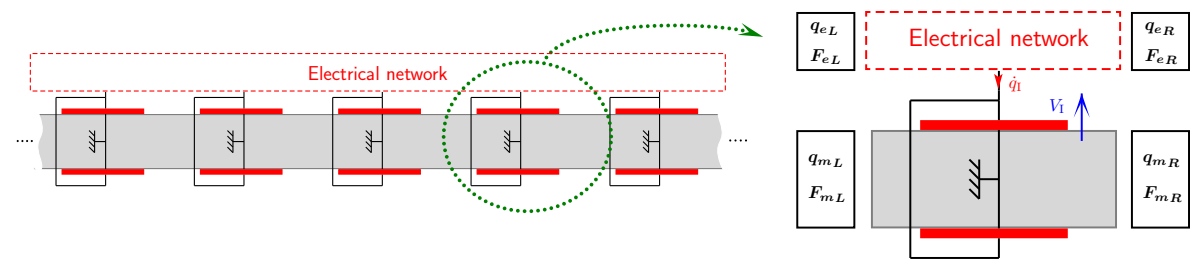

Figure 1: Interconnected array of piezoelectric patches and corresponding unit cell with left and right electromechanical degrees of freedom.

where the vector $\boldsymbol{q}_{\boldsymbol{m}}$ corresponds to the nodal values of $u_{i}$. The matrices $\boldsymbol{M}_{\boldsymbol{m}}, \boldsymbol{K}_{\boldsymbol{m}}$ and $\boldsymbol{K}_{\boldsymbol{c}}$ are respectively the mass, stiffness and coupling matrices and $\boldsymbol{F}_{\boldsymbol{m}}$ corresponds to the mechanical forcing. In the end, the general finite element formulation of the electromechanical problem can be expressed as

$$
\left[\begin{array}{cc}
\boldsymbol{M}_{\boldsymbol{m}} & \mathbf{0} \\
\mathbf{0} & 0
\end{array}\right]\left[\begin{array}{c}
\ddot{\boldsymbol{q}}_{\boldsymbol{m}} \\
\ddot{V}_{\mathrm{I}}
\end{array}\right]+\left[\begin{array}{cc}
\boldsymbol{K}_{\boldsymbol{m}} & \boldsymbol{K}_{\boldsymbol{c}} \\
-\boldsymbol{K}_{\boldsymbol{c}} \boldsymbol{T} & C^{\varepsilon}
\end{array}\right]\left[\begin{array}{c}
\boldsymbol{q}_{\boldsymbol{m}} \\
V_{\mathrm{I}}
\end{array}\right]=\left[\begin{array}{c}
\boldsymbol{F}_{\boldsymbol{m}} \\
q_{\mathrm{I}}
\end{array}\right] .
$$

\subsection{Piezoelectric unit cell}

Our objective is now to apply the previous FE model to the unit cell of an electromechanical waveguide as the one represented in Fig. 1], where an electrical network is coupled to a mechanical structure through an array of piezoelectric elements. Each unit cell involves a pair of piezoelectric patches connected in parallel. Other geometries or electrical connections could be considered but the analysis is here restrained to a single piezoelectric degree of freedom per unit cell. Since $\dot{q}_{\mathrm{I}}=\mathrm{j} \omega q_{\mathrm{I}}$ represents the electrical current flowing through the pair of piezoelectric patches at angular frequency $\omega$, a shunt made of an impedance $Z$ in parallel with the piezoelectric elements gives the relation $V_{\mathrm{I}}=-\mathrm{j} \omega Z q_{\mathrm{I}}$, which allows to condense the electrical degrees of freedom. Indeed, under harmonic forcing, it is obtained from Eq. (9) that

$$
\left[\boldsymbol{K}_{\boldsymbol{m}}+\frac{1}{C^{\varepsilon}+\frac{1}{j \omega Z}} \boldsymbol{K}_{c} \boldsymbol{K}_{\boldsymbol{c}} \boldsymbol{T}^{\boldsymbol{T}}-\omega^{2} \boldsymbol{M}_{\boldsymbol{m}}\right] \boldsymbol{q}_{\boldsymbol{m}}=\boldsymbol{F}_{\boldsymbol{m}},
$$

which represents a standard mechanical problem with an added-stiffness term due to piezoelectric coupling 22, 24].

However, this method is not applicable when considering interconnections of successive unit cells with an electrical network. There are electrical charges that are flowing in and out of the unit cell. This means that there is no direct relation between the internal variables $V_{\mathrm{I}}$ and $q_{\mathrm{I}}$. In the end, the electrical variables cannot be condensed in the mechanical problem. A novel formulation that is able to take into account external electrical degrees of freedom is thus required.

\subsection{Electrical and mechanical degrees of freedom}

From the topology of the electrical network, the electric charge $q_{\mathrm{I}}$ flowing through the pair of piezoelectric patches can be defined relatively to a charge vector $\boldsymbol{q}_{\boldsymbol{e}}$ as

$$
q_{\mathrm{I}}=S^{T} q_{e}
$$

Focusing on a one-dimensional unit cell, the vector $\boldsymbol{q}_{\boldsymbol{e}}=\left[\begin{array}{l}\boldsymbol{q}_{\boldsymbol{e} \mathrm{L}} \\ \boldsymbol{q}_{\boldsymbol{e} \mathrm{R}}\end{array}\right]^{T}$ refers to the electrical charges that are exchanged with the neighboring left and right unit cells, while $\boldsymbol{S}$ depends on the internal connections of the considered portion of electrical network. By analogy with the force vector $\boldsymbol{F}_{\boldsymbol{m}}$, we define $\boldsymbol{F}_{\boldsymbol{e}}=\left[\begin{array}{ll}\boldsymbol{F}_{\boldsymbol{e} \mathrm{L}} & \boldsymbol{F}_{\boldsymbol{e} \mathrm{R}}\end{array}\right]^{T}$ that contains the voltage contributions on both sides of the electrical part of the unit cell. In the following, we consider that the total energy of the purely electrical problem (when $\boldsymbol{q}_{\boldsymbol{m}}=\mathbf{0}$ ) can be written under the form

$$
E_{e}=\frac{1}{2} \boldsymbol{q}_{e}^{\boldsymbol{T}} \boldsymbol{K}_{e} \boldsymbol{q}_{e}+\frac{1}{2} \dot{\boldsymbol{q}}_{e}^{\boldsymbol{T}} \boldsymbol{M}_{e} \dot{\boldsymbol{q}}_{e}
$$


where $\boldsymbol{K}_{\boldsymbol{e}}$ and $\boldsymbol{M}_{\boldsymbol{e}}$ are symmetric and positive definite matrices that are the electrical analogues of mechanical stiffness and mass matrices. From this formulation, the total energy in an electrical network made of passive components, such as capacitors and inductors, is clearly represented as the sum of two contributions: $E_{e}=E_{e}^{C}+E_{e}^{L}$, where $E_{e}^{C}$ corresponds to the potential energy stored in the capacitors and $E_{e}^{L}$ corresponds to the magnetic energy stored in the inductors. This highlights the direct electromechanical analogy between a spring and a capacitor and between a mass and an inductors [41, which also induces that $E_{e}^{C}$ and $E_{e}^{L}$ are analogous to elastic and kinetic energies, respectively.

The energy in the electrical network excluding the piezoelectric capacitor is $E_{e}-\frac{q_{\mathrm{I}}^{2}}{2 C^{\varepsilon}}$. Furthermore, the electrical power flowing into the electrical network in Fig. 1 is equal to

$$
P_{e}=\boldsymbol{F}_{\boldsymbol{e}}^{\boldsymbol{T}} \dot{\boldsymbol{q}}_{\boldsymbol{e}}-V_{\mathrm{I}} \dot{q}_{\mathrm{I}}
$$

If the electrical network is a conservative system, a power balance induces that the input power is equal to the time derivative of the energy as

$$
P_{e}=\frac{d\left(E_{e}-\frac{q_{\mathrm{I}}^{2}}{2 C^{\varepsilon}}\right)}{d t} .
$$

The symmetry property of both matrices $\boldsymbol{K}_{\boldsymbol{e}}$ and $\boldsymbol{M}_{\boldsymbol{e}}$ thus gives

$$
\boldsymbol{F}_{e}^{\boldsymbol{T}} \dot{\boldsymbol{q}}_{e}-V_{\mathrm{I}} \dot{q}_{\mathrm{I}}=\boldsymbol{q}_{e}^{\boldsymbol{T}} \boldsymbol{K}_{e} \dot{\boldsymbol{q}}_{e}+\dot{\boldsymbol{q}}_{\boldsymbol{e}}^{\boldsymbol{T}} \boldsymbol{M}_{e} \ddot{\boldsymbol{q}}_{e}-\frac{q_{\mathrm{I}}}{C^{\varepsilon}} \dot{q}_{\mathrm{I}} .
$$

Considering Eq. (11), this is equivalent to

$$
\dot{\boldsymbol{q}}_{\boldsymbol{e}}^{\boldsymbol{T}}\left(\boldsymbol{F}_{\boldsymbol{e}}-\boldsymbol{S} V_{\mathrm{I}}-\boldsymbol{K}_{\boldsymbol{e}} \boldsymbol{q}_{\boldsymbol{e}}-\boldsymbol{M}_{\boldsymbol{e}} \ddot{\boldsymbol{q}}_{\boldsymbol{e}}+\frac{1}{C^{\varepsilon}} \boldsymbol{S} \boldsymbol{S}^{\boldsymbol{T}} \boldsymbol{q}_{\boldsymbol{e}}\right)=0,
$$

which is true for any electric current vector $\dot{\boldsymbol{q}}_{e}$ so

$$
\boldsymbol{F}_{\boldsymbol{e}}=\boldsymbol{K}_{\boldsymbol{e}} \boldsymbol{q}_{\boldsymbol{e}}+\boldsymbol{M}_{\boldsymbol{e}} \ddot{\boldsymbol{q}}_{\boldsymbol{e}}+\boldsymbol{S} V_{\mathrm{I}}-\frac{1}{C^{\varepsilon}} \boldsymbol{S} \boldsymbol{S}^{\boldsymbol{T}} \boldsymbol{q}_{\boldsymbol{e}}
$$

In the end, as Eq. (9) also gives

$$
V_{\mathrm{I}}=\frac{1}{C^{\varepsilon}}\left(\boldsymbol{S}^{\boldsymbol{T}} \boldsymbol{q}_{\boldsymbol{e}}+\boldsymbol{K}_{\boldsymbol{c}}^{\boldsymbol{T}} \boldsymbol{q}_{\boldsymbol{m}}\right),
$$

it is obtained a relation between the voltage vector $\boldsymbol{F}_{\boldsymbol{e}}$ and the displacement vectors:

$$
\boldsymbol{F}_{\boldsymbol{e}}=\boldsymbol{K}_{\boldsymbol{e}} \boldsymbol{q}_{\boldsymbol{e}}+\boldsymbol{M}_{\boldsymbol{e}} \ddot{\boldsymbol{q}}_{\boldsymbol{e}}+\frac{1}{C^{\varepsilon}} \boldsymbol{S} \boldsymbol{K}_{\boldsymbol{c}}^{\boldsymbol{T}} \boldsymbol{q}_{\boldsymbol{m}}
$$

Note that a principle of superposition applies because $\boldsymbol{F}_{\boldsymbol{e}}$ is a sum of two contributions. The first one corresponds to the voltage vector when $\boldsymbol{q}_{\boldsymbol{m}}=\mathbf{0}$, i.e. when no mechanical displacement is allowed. This is a purely electrical contribution that only depends on the electrical network and the piezoelectric capacitance. On the other hand, the second contribution, observed when $\boldsymbol{q}_{\boldsymbol{e}}=\mathbf{0}$, is directly related to piezoelectric coupling.

Now that all the mechanical and electrical vectors have been defined for both displacements and forcing terms, they can be organized into a single matrix formulation. Under harmonic excitation at angular frequency $\omega$, Eqs. (9) and (18) lead to a force vector

$$
\boldsymbol{F}_{\boldsymbol{m}}=\left[\boldsymbol{K}_{\boldsymbol{m}}+\frac{1}{C^{\varepsilon}} \boldsymbol{K}_{\boldsymbol{c}} \boldsymbol{K}_{\boldsymbol{c}}^{\boldsymbol{T}}-\omega^{2} \boldsymbol{M}_{\boldsymbol{m}}\right] \boldsymbol{q}_{\boldsymbol{m}}+\frac{1}{C^{\varepsilon}} \boldsymbol{K}_{\boldsymbol{c}} \boldsymbol{S}^{\boldsymbol{T}} \boldsymbol{q}_{\boldsymbol{e}}
$$

Similarly, Eq. (19) gives a voltage vector

$$
\boldsymbol{F}_{\boldsymbol{e}}=\left[\boldsymbol{K}_{\boldsymbol{e}}-\omega^{2} \boldsymbol{M}_{\boldsymbol{e}}\right] \boldsymbol{q}_{\boldsymbol{e}}+\frac{1}{C^{\varepsilon}} \boldsymbol{S} \boldsymbol{K}_{\boldsymbol{c}}^{\boldsymbol{T}} \boldsymbol{q}_{\boldsymbol{m}}
$$


This brings the following DSM formulation involving a combination of mechanical and electrical degrees of freedom:

$$
\left[\left[\begin{array}{cc}
\boldsymbol{K}_{\boldsymbol{m}}+\frac{1}{C^{\varepsilon}} \boldsymbol{K}_{\boldsymbol{c}} \boldsymbol{K}_{\boldsymbol{c}}^{\boldsymbol{T}} & \frac{1}{C^{\varepsilon}} \boldsymbol{K}_{\boldsymbol{c}} \boldsymbol{S}^{\boldsymbol{T}} \\
\frac{1}{C^{\varepsilon}} \boldsymbol{S} \boldsymbol{K}_{\boldsymbol{c}}^{\boldsymbol{T}} & \boldsymbol{K}_{\boldsymbol{e}}
\end{array}\right]-\omega^{2}\left[\begin{array}{cc}
\boldsymbol{M}_{\boldsymbol{m}} & \mathbf{0} \\
\mathbf{0} & \boldsymbol{M}_{\boldsymbol{e}}
\end{array}\right]\right]\left[\begin{array}{c}
\boldsymbol{q}_{\boldsymbol{m}} \\
\boldsymbol{q}_{\boldsymbol{e}}
\end{array}\right]=\left[\begin{array}{c}
\boldsymbol{F}_{\boldsymbol{m}} \\
\boldsymbol{F}_{\boldsymbol{e}}
\end{array}\right]
$$

The coupled problem is thus organized like any purely mechanical problem involving mass and stiffness symmetric matrices. The only difference is that the global displacement and force vectors contain both mechanical and electrical contributions. With such an electromechanical formulation, classical methods as the assembly of element matrices or TM methods can be directly applied.

\section{Wave Finite Element method}

Since a unit cell that involves coupling to an electrical network can be described by the equivalent of a DSM, the WFE method can be used to compute propagation constants and frequency response functions. Focusing on the case of an electromechanical waveguide, the main results of the WFE method are recalled and the TM that is built from a FE formulation is then combined with the Riccati transfer matrix (RTM) method as an alternative to solve numerical issues.

\subsection{From a dynamic stiffness matrix to a transfer matrix}

The DSM given in Eq. 22) is rearranged to bring together the mechanical and electrical left and right degrees of freedom [29], as

$$
\left[\begin{array}{ccc}
\tilde{\boldsymbol{D}}_{\mathrm{LL}} & \tilde{\boldsymbol{D}}_{\mathrm{LI}} & \tilde{\boldsymbol{D}}_{\mathrm{LR}} \\
\tilde{\boldsymbol{D}}_{\mathrm{IL}} & \tilde{\boldsymbol{D}}_{\mathrm{II}} & \tilde{\boldsymbol{D}}_{\mathrm{IR}} \\
\tilde{\boldsymbol{D}}_{\mathrm{RL}} & \tilde{\boldsymbol{D}}_{\mathrm{RI}} & \tilde{\boldsymbol{D}}_{\mathrm{RR}}
\end{array}\right]\left[\begin{array}{c}
\boldsymbol{q}_{\mathrm{L}} \\
\boldsymbol{q}_{\boldsymbol{m}_{\mathrm{I}}} \\
\boldsymbol{q}_{\mathrm{R}}
\end{array}\right]=\left[\begin{array}{c}
\boldsymbol{F}_{\mathrm{L}} \\
\mathbf{0} \\
\boldsymbol{F}_{\mathrm{R}}
\end{array}\right],
$$

where $\boldsymbol{q}_{\mathrm{L}}=\left[\begin{array}{ll}\boldsymbol{q}_{\boldsymbol{m} \mathrm{L}} & \boldsymbol{q}_{\boldsymbol{e} \mathrm{L}}\end{array}\right]^{T}, \boldsymbol{q}_{\mathrm{R}}=\left[\begin{array}{ll}\boldsymbol{q}_{\boldsymbol{m} \mathrm{R}} & \boldsymbol{q}_{\boldsymbol{e} \mathrm{R}}\end{array}\right]^{T}, \boldsymbol{F}_{\mathrm{L}}=\left[\begin{array}{ll}\boldsymbol{F}_{\boldsymbol{m}_{\mathrm{L}}} & \boldsymbol{F}_{\boldsymbol{e} \mathrm{L}}\end{array}\right]^{T}$ and $\boldsymbol{F}_{\mathrm{R}}=\left[\begin{array}{ll}\boldsymbol{F}_{\boldsymbol{m} \mathrm{R}} & \boldsymbol{F}_{\boldsymbol{e} \mathrm{R}}\end{array}\right]^{T}$. This expression is based on the decomposition of the mechanical vectors $\boldsymbol{F}_{\boldsymbol{m}}=\left[\begin{array}{lll}\boldsymbol{F}_{\boldsymbol{m} \mathrm{L}} & \mathbf{0} & \boldsymbol{F}_{\boldsymbol{m} \mathrm{R}}\end{array}\right]^{T}$ and $\boldsymbol{q}_{\boldsymbol{m}}=$ $\left[\begin{array}{lll}\boldsymbol{q}_{\boldsymbol{m}_{\mathrm{L}}} & \boldsymbol{q}_{\boldsymbol{m}_{\mathrm{I}}} \boldsymbol{q}_{\boldsymbol{m}_{\mathrm{R}}}\end{array}\right]^{T}$ where $\boldsymbol{q}_{\boldsymbol{m}_{\mathrm{I}}}$ is the mechanical displacement vector of the internal nodes of the unit cell. A similar form applies in the electrical domain as $\boldsymbol{F}_{\boldsymbol{e}}=\left[\begin{array}{ll}\boldsymbol{F}_{\boldsymbol{e} \mathrm{L}} & \boldsymbol{F}_{\boldsymbol{e} \mathrm{R}}\end{array}\right]^{T}$ and $\boldsymbol{q}_{\boldsymbol{e}}=\left[\begin{array}{ll}\boldsymbol{q}_{\boldsymbol{e} \mathrm{L}} & \boldsymbol{q}_{\boldsymbol{e} \mathrm{R}}\end{array}\right]^{T}$. Note that no internal nodes have been represented in the electrical vectors because they are directly condensed in the following example involving a limited number of electrical components. Yet, for more complicated networks, internal electrical degrees of freedom can be initially retained such as for the mechanical part. With this partitioning of the electromechanical vectors, the procedures of the WFE method [18 23] can be implemented. This starts with an elimination of the internal degrees of freedoms through

$$
\left[\begin{array}{ll}
\boldsymbol{D}_{\mathrm{LL}} & \boldsymbol{D}_{\mathrm{LR}} \\
\boldsymbol{D}_{\mathrm{RL}} & \boldsymbol{D}_{\mathrm{RR}}
\end{array}\right]\left[\begin{array}{c}
\boldsymbol{q}_{\mathrm{L}} \\
\boldsymbol{q}_{\mathrm{R}}
\end{array}\right]=\left[\begin{array}{c}
\boldsymbol{F}_{\mathrm{L}} \\
\boldsymbol{F}_{\mathrm{R}}
\end{array}\right]
$$

where

$$
\begin{aligned}
& \boldsymbol{D}_{\mathrm{LL}}=\tilde{\boldsymbol{D}}_{\mathrm{LL}}-\tilde{\boldsymbol{D}}_{\mathrm{LI}} \tilde{\boldsymbol{D}}_{\mathrm{II}}^{-1} \tilde{\boldsymbol{D}}_{\mathrm{IL}} \\
& \boldsymbol{D}_{\mathrm{LR}}=\tilde{\boldsymbol{D}}_{\mathrm{LR}}-\tilde{\boldsymbol{D}}_{\mathrm{LI}} \tilde{\boldsymbol{D}}_{\mathrm{II}}^{-1} \tilde{\boldsymbol{D}}_{\mathrm{IR}} \\
& \boldsymbol{D}_{\mathrm{RL}}=\tilde{\boldsymbol{D}}_{\mathrm{RL}}-\tilde{\boldsymbol{D}}_{\mathrm{RI}} \tilde{\boldsymbol{D}}_{\mathrm{II}}^{-\mathbf{1}} \tilde{\boldsymbol{D}}_{\mathrm{IL}} \\
& \boldsymbol{D}_{\mathrm{RR}}=\tilde{\boldsymbol{D}}_{\mathrm{RR}}-\tilde{\boldsymbol{D}}_{\mathrm{RI}} \tilde{\boldsymbol{D}}_{\mathrm{II}}^{-\mathbf{1}} \tilde{\boldsymbol{D}}_{\mathrm{IR}}
\end{aligned}
$$

and the condensed DSM is then transformed into a TM:

$$
\left[\begin{array}{c}
\boldsymbol{q}_{\mathrm{R}} \\
\boldsymbol{F}_{\mathrm{R}}
\end{array}\right]=\left[\begin{array}{cc}
-\boldsymbol{D}_{\mathrm{LR}}^{-1} \boldsymbol{D}_{\mathrm{LL}} & -\boldsymbol{D}_{\mathrm{LR}}^{-1} \\
\boldsymbol{D}_{\mathrm{RL}}-\boldsymbol{D}_{\boldsymbol{R} \boldsymbol{R}} \boldsymbol{D}_{\mathrm{LR}}^{-1} \boldsymbol{D}_{\mathrm{LL}} & -\boldsymbol{D}_{\mathrm{RR}} \boldsymbol{D}_{\mathrm{LR}}^{-1}
\end{array}\right]\left[\begin{array}{c}
\boldsymbol{q}_{\mathrm{L}} \\
-\boldsymbol{F}_{\mathrm{L}}
\end{array}\right]
$$

As both force vectors $\boldsymbol{F}_{\mathrm{L}}$ and $\boldsymbol{F}_{\mathrm{R}}$ corresponds to external forcing, the use of $-\boldsymbol{F}_{\mathrm{L}}$ is required in order to ensure the continuity of the state vector [18]. 


\subsection{Propagation constants and frequency response functions}

A one-dimensional periodic structure can be defined as a series of identical unit cells. As in Eq. (26), it is recalled that the relation between the states at the right and left ends of a unit cell is described by a TM called $\boldsymbol{T}$ through

$$
\left[\begin{array}{c}
\boldsymbol{q}_{\mathrm{R}} \\
\boldsymbol{F}_{\mathrm{R}}
\end{array}\right]=\boldsymbol{T}\left[\begin{array}{c}
\boldsymbol{q}_{\mathrm{L}} \\
-\boldsymbol{F}_{\mathrm{L}}
\end{array}\right]
$$

The Floquet-Bloch theory induces that, for a wave propagating in the one-dimensional medium, the state on the right of a unit cell is equal to $e^{ \pm \mu}$ times the state on the left [17, the sign depending on the direction of the wave,

$$
\left[\begin{array}{c}
\boldsymbol{q}_{\mathrm{R}} \\
\boldsymbol{F}_{\mathrm{R}}
\end{array}\right]=e^{ \pm \mu}\left[\begin{array}{c}
\boldsymbol{q}_{\mathrm{L}} \\
-\boldsymbol{F}_{\mathrm{L}}
\end{array}\right] \text {. }
$$

Here, $\mu$ is the propagation constant, which is usually defined as $\mu=\delta+\mathrm{j} \theta$, where $\delta$ is the attenuation constant, $\mathrm{j}$ is the imaginary unit and $\theta$ is the phase constant [7. Consequently, if $\left[\boldsymbol{q}_{\mathrm{L}}-\boldsymbol{F}_{\mathrm{L}}\right]^{T}$ is an eigenvector of the TM, then $\mu=\ln (\lambda)$, where $\lambda$ represents an eigenvalue of $\boldsymbol{T}$ :

$$
\boldsymbol{T}\left[\begin{array}{c}
\boldsymbol{q}_{\mathrm{L}} \\
-\boldsymbol{F}_{\mathrm{L}}
\end{array}\right]=\lambda\left[\begin{array}{c}
\boldsymbol{q}_{\mathrm{L}} \\
-\boldsymbol{F}_{\mathrm{L}}
\end{array}\right] .
$$

As mentioned by Mace et al. [18, although the solution of this eigenvalue problem is straightforward when the number of degrees of freedom is small (as in the following examples), more complicated cases often require the use of alternative eigenvalue schemes to prevent numerical ill-conditioning [19 21, 23, 42]. In any case, the eigensolution can be written under the form

$$
T \Phi=\Phi \Lambda,
$$

where $\boldsymbol{\Lambda}$ is a diagonal matrix containing the eigenvalues and $\boldsymbol{\Phi}$ is build from the corresponding eigenvectors that represent wave shapes. Considering state vectors including both mechanical and electrical variables, we obtain a set of wave modes $\{\boldsymbol{\Lambda}, \boldsymbol{\Phi}\}$ related to propagation in an electromechanical waveguide. Generally speaking, we cannot distinguish mechanical waves from electrical waves because of the coupling between both physical domains. This highlights the necessity of the proposed unified formulation combining mechanical and electrical degrees of freedom.

Apart from propagation constants and waveshapes, the TM model enables computation of frequency response functions. According to the definition of a periodic structure, each unit cell is described by an identical TM. Consequently, a basic solution to obtain the mechanical state on the right of the $n^{\text {th }}$ unit cell consists in raising the corresponding TM to the power of $n$. From Eq. (30), it is obtained

$$
\left[\begin{array}{c}
q_{n} \\
F_{n}
\end{array}\right]=\Phi \Lambda^{n} \Phi^{-1}\left[\begin{array}{c}
q_{0} \\
-F_{0}
\end{array}\right]=\left[\begin{array}{ll}
T_{n}^{q q} & T_{n}^{q F} \\
T_{n}^{F q} & T_{n}^{F F}
\end{array}\right]\left[\begin{array}{c}
q_{0} \\
-F_{0}
\end{array}\right],
$$

where the subscript 0 refers to the left end of the first unit cell and the forcing vectors corresponds to forces or voltages applied to the considered electromechanical structure. Then, boundary conditions need to be introduced. For example, with a prescribed force vector $\boldsymbol{F}_{\mathbf{0}}$ applied to the left end of a free-free periodic structure made of $n$ unit cells, as $\boldsymbol{F}_{\boldsymbol{n}}=\mathbf{0}$, the displacement $\boldsymbol{q}_{\boldsymbol{n}}$ at the right end is defined from Eq. (31) as

$$
q_{n}=\left(T_{n}^{q q} T_{n}^{F q^{-1}} T_{n}^{F F}-T_{n}^{q F}\right) F_{0} .
$$

Similar formulations can be used for other boundary conditions such as free or clamped for the mechanical part and short-circuited or open-circuited for the electrical part. In any case, it becomes possible to define frequency response functions between any variables at both ends of the electromechanical structure.

Equation (31) represents one of the simplest solution to obtain the relation between the state variables at the ends of the periodic structure. However, such a two-point boundary value problem is ill-conditioned [35 38, which can lead to numerical instabilities. The deficiency of Eq. 32 is confirmed in Fig. 2 when 


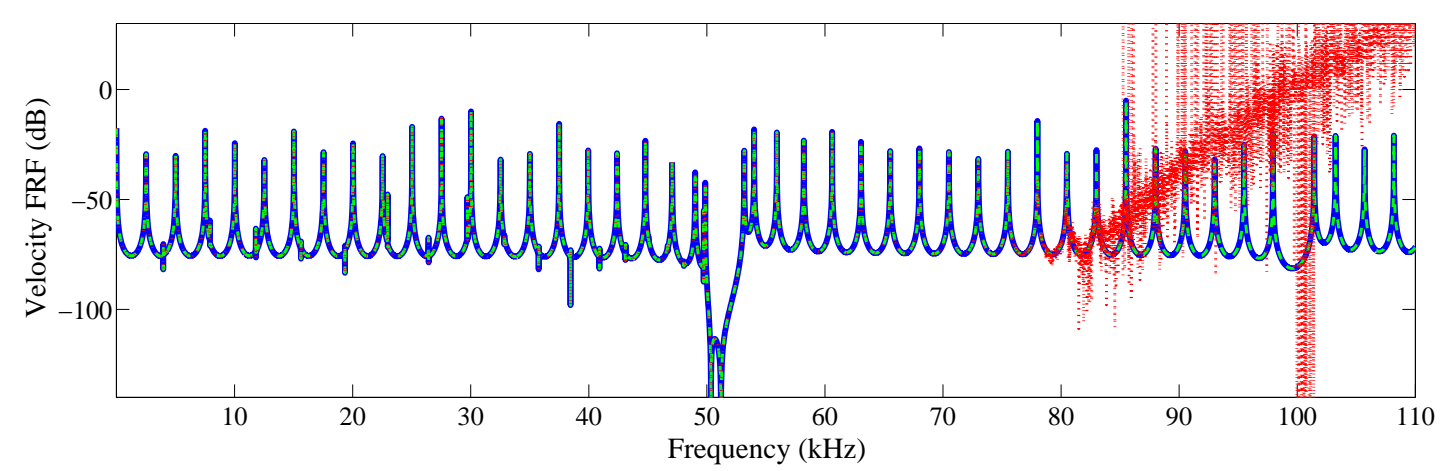

Figure 2: Comparison of computational methods - ( . .) transfer matrix (TM) method based on Eq. 32 , ( -$)$ dynamic stiffness matrix (DSM) approach based on Eq. 34 and (--) Riccati transfer matrix (RTM) method.

computing frequency response functions after nondimensionalization of the state vectors. Based the first example proposed in the next section, numerical errors seriously affect the computation above $80 \mathrm{kHz}$. Still, several solutions have been proposed to compute forced responses from the WFE method [19 21]. A convenient approach consists in the definition of a global DSM $\boldsymbol{D}_{\boldsymbol{n}}$ that relates displacements and forces at both ends of the finite structure through

$$
\left[\begin{array}{l}
F_{0} \\
F_{n}
\end{array}\right]=D_{n}\left[\begin{array}{c}
q_{0} \\
q_{n}
\end{array}\right]
$$

Silva et al. 21 have shown that the DSM can be expressed as

$$
D_{n}=\left[\begin{array}{cc}
-\Phi_{F} & -\boldsymbol{\Phi}_{F}^{\star} \lambda^{n} \\
\boldsymbol{\Phi}_{F} \lambda^{n} & \boldsymbol{\Phi}_{F}^{\star}
\end{array}\right]\left[\begin{array}{cc}
\mathbf{I} & \boldsymbol{\Phi}_{q}^{-1} \boldsymbol{\Phi}_{q}^{\star} \lambda^{n} \\
\boldsymbol{\Phi}_{q}^{\star-1} \boldsymbol{\Phi}_{q} \lambda^{n} & \mathbf{I}
\end{array}\right]\left[\begin{array}{cc}
\boldsymbol{\Phi}_{q}^{-1} & 0 \\
0 & \boldsymbol{\Phi}_{q}^{\star-1}
\end{array}\right],
$$

where the square matrices $\Phi_{\boldsymbol{q}}, \boldsymbol{\Phi}_{\boldsymbol{q}}^{\star}, \boldsymbol{\Phi}_{\boldsymbol{F}}, \boldsymbol{\Phi}_{\boldsymbol{F}}^{\star}$ and $\boldsymbol{\lambda}$ come from a wave basis that is organized in two distinct sets of right-going and left-going waves (without superscript and with $\star$ superscript, respectively):

$$
\boldsymbol{\Phi}=\left[\begin{array}{cc}
\boldsymbol{\Phi}_{q} & \boldsymbol{\Phi}_{q}^{\star} \\
\boldsymbol{\Phi}_{F} & \boldsymbol{\Phi}_{F}^{\star}
\end{array}\right] \text { and } \boldsymbol{\Lambda}=\left[\begin{array}{cc}
\lambda & 0 \\
0 & \lambda^{\star}
\end{array}\right]
$$

The results obtained with this DSM approach are shown in Fig. 2 which highlights the numerical stablility of the formulation in Ref. 21]. Yet, note that the DSM proposed by Duhamel et al. [19] had first been used for the computation but numerical issues similar to that obtained from Eq. 32 were observed from $80 \mathrm{kHz}$.

\subsection{Riccati transfer matrix method}

Alternatively, Horner and Pilkey proposed a numerically reliable formulation based on a RTM method 35 38. This method is presented below for a case where external forces and voltages only apply at one of the two ends of the electromechanical periodic structure. First, the TM is partitioned as

$$
\left[\begin{array}{c}
q_{i+1} \\
\boldsymbol{F}_{i+1}
\end{array}\right]=\left[\begin{array}{cc}
\boldsymbol{T}^{q \boldsymbol{q}} & \boldsymbol{T}^{\boldsymbol{q} F} \\
\boldsymbol{T}^{\boldsymbol{F q}} & \boldsymbol{T}^{\boldsymbol{F F}}
\end{array}\right]\left[\begin{array}{c}
\boldsymbol{q}_{i} \\
\boldsymbol{F}_{i}
\end{array}\right]
$$

where the subscript $i$ goes from 0 to $n-1$ when the periodic structure is made of $n$ unit cells. The procedure relies on the Riccati transform, which postulates that the forces and the displacements at both ends of a unit cell are given by

$$
\begin{aligned}
& \boldsymbol{F}_{i+1}=\boldsymbol{R}_{i+1} \boldsymbol{q}_{i+1}+\boldsymbol{g}_{i+1}, \\
& \boldsymbol{F}_{i}=\boldsymbol{R}_{i} \boldsymbol{q}_{i}+\boldsymbol{g}_{i}
\end{aligned}
$$


where the Riccati matrix $\boldsymbol{R}$ and the column vector of force components $\boldsymbol{g}$ are to be determined. To this end, a recursive scheme is implemented. From Eqs. (36) and (37), it is found that

$$
\begin{aligned}
& \boldsymbol{q}_{i+1}=\boldsymbol{T}^{\boldsymbol{q q}} \boldsymbol{q}_{i}+\boldsymbol{T}^{\boldsymbol{q} \boldsymbol{F}}\left[\boldsymbol{R}_{i} \boldsymbol{q}_{i}+\boldsymbol{g}_{i}\right] \\
& \boldsymbol{R}_{i+1} \boldsymbol{q}_{i+1}+\boldsymbol{g}_{i+1}=\boldsymbol{T}^{\boldsymbol{F} \boldsymbol{q}} \boldsymbol{q}_{i}+\boldsymbol{T}^{\boldsymbol{F} \boldsymbol{F}}\left[\boldsymbol{R}_{i} \boldsymbol{q}_{i}+\boldsymbol{g}_{i}\right]
\end{aligned}
$$

which leads to

$$
\left(R_{i+1}\left[\boldsymbol{T}^{q q}+\boldsymbol{T}^{q \boldsymbol{F}} \boldsymbol{R}_{i}\right]-\left[\boldsymbol{T}^{\boldsymbol{F q}}+\boldsymbol{T}^{\boldsymbol{F} \boldsymbol{F}} \boldsymbol{R}_{i}\right]\right) \boldsymbol{q}_{i}+\left(g_{i+1}+\left[\boldsymbol{R}_{i+1} \boldsymbol{T}^{q \boldsymbol{F}}-\boldsymbol{T}^{\boldsymbol{F} \boldsymbol{F}}\right] g_{i}\right)=\mathbf{0} .
$$

In order to have Eq. (39) true for any arbitrary $\boldsymbol{q}_{i}$, it is required that

$$
\begin{aligned}
& \boldsymbol{g}_{i+1}+\left[\boldsymbol{R}_{i+1} \boldsymbol{T}^{\boldsymbol{q} \boldsymbol{F}}-\boldsymbol{T}^{\boldsymbol{F} \boldsymbol{F}}\right] \boldsymbol{g}_{i}=\mathbf{0} \\
& \boldsymbol{R}_{i+1}\left[\boldsymbol{T}^{q \boldsymbol{q}}+\boldsymbol{T}^{\boldsymbol{q} \boldsymbol{F}} \boldsymbol{R}_{i}\right]-\left[\boldsymbol{T}^{\boldsymbol{F} \boldsymbol{q}}+\boldsymbol{T}^{\boldsymbol{F} \boldsymbol{F}} \boldsymbol{R}_{i}\right]=\mathbf{0}
\end{aligned},
$$

which can be reformulated as

$$
\begin{aligned}
& \boldsymbol{g}_{i}=\left[\boldsymbol{T}^{\boldsymbol{F} \boldsymbol{F}}-\boldsymbol{R}_{i+1} \boldsymbol{T}^{\boldsymbol{q} \boldsymbol{F}}\right]^{-1} \boldsymbol{g}_{i+1} \\
& \boldsymbol{R}_{i}=\left[\boldsymbol{T}^{\boldsymbol{F} F}-\boldsymbol{R}_{i+1} \boldsymbol{T}^{q \boldsymbol{F}}\right]^{-1}\left[\boldsymbol{R}_{i+1} \boldsymbol{T}^{q \boldsymbol{q}}-\boldsymbol{T}^{\boldsymbol{F} q}\right]
\end{aligned}
$$

Eqs. (41) represents the "backward in space" recursive process 38] based on $n$ iterations, which compute all $\boldsymbol{g}_{i}$ and $\boldsymbol{R}_{i}$ from the knowledge of their values at the right end of the structure, i.e. $\boldsymbol{g}_{n}$ and $\boldsymbol{R}_{n}$. Then, the displacement and force vectors can be computed all along the structure with the "forward in space" process which still involves $n$ iterations deduced from Eqs. (37) and (38) as

$$
\begin{aligned}
& \boldsymbol{q}_{i+1}=\left[\boldsymbol{T}^{\boldsymbol{q} \boldsymbol{q}}+\boldsymbol{T}^{\boldsymbol{q} \boldsymbol{F}} \boldsymbol{R}_{i}\right] \boldsymbol{q}_{i}+\boldsymbol{T}^{\boldsymbol{q} \boldsymbol{F}} \boldsymbol{g}_{i} \\
& \boldsymbol{F}_{i+1}=\boldsymbol{R}_{i+1} \boldsymbol{q}_{i+1}+\boldsymbol{g}_{i+1}
\end{aligned}
$$

For example, because $\boldsymbol{F}_{\boldsymbol{n}}=\mathbf{0}$ at the non-excited end of a free-free periodic structure and $\boldsymbol{F}_{n}=\boldsymbol{R}_{n} \boldsymbol{q}_{n}+\boldsymbol{g}_{n}$, one can set $\boldsymbol{R}_{n}=\mathbf{0}$ and $\boldsymbol{g}_{n}=\mathbf{0}$. Furthermore, with a prescribed force $\boldsymbol{F}_{\mathbf{0}}$ applied to the left end, $\boldsymbol{q}_{0}=$ $\boldsymbol{R}_{0}{ }^{-1}\left[\boldsymbol{F}_{0}-\boldsymbol{g}_{0}\right]$ and the displacement $\boldsymbol{q}_{\boldsymbol{n}}$ at the right end is then computed from Eqs. 41 and $(42)$. Figure 2 shows the efficiency of this method compared to the use of the ill-conditioned Eq. (32). As when using the DSM approach in Eq. (34) which gives similar results, no numerical issues have been observed when increasing the upper limit frequency or the number of unit cells, which highlights the interest of the RTM method for the analysis of periodic electromechanical waveguides. Also note that the method is not limited to matrices built from a FE procedure. If available, analytic TM [32, 33. can obviously be used for similar computations.

\section{Application to interconnected piezoelectric networks}

Both the WFE method and the RTM method are applied to electromechanical waveguides involving a rod and a beam covered with an array of piezoelectric patches. Second and forth order transmission lines interconnect the patches, thus allowing wave propagation through electrical media and subsequent alteration of the dynamic properties of the whole coupled structure.

\subsection{Rod coupled to a second order transmission line}

The case of a rod subjected to longitudinal propagation is first considered. As show in Fig. 3 , the rod is covered with an array of periodic patches which are interconnected through a line of inductors $L$. This build a second order transmission line [43, that is the discrete analogue of a rod [25, 44. Focusing on the mechanical part of the problem, linear shape functions applied to a rod element 24] lead to the following element stiffness and mass matrices:

$$
\boldsymbol{K}_{\boldsymbol{m}}^{\mathrm{e}}=\frac{Y S}{a_{e}}\left[\begin{array}{cc}
1 & -1 \\
-1 & 1
\end{array}\right] \quad \text { and } \quad \boldsymbol{M}_{\boldsymbol{m}}^{\mathrm{e}}=\frac{\rho S a_{e}}{6}\left[\begin{array}{ll}
2 & 1 \\
1 & 2
\end{array}\right],
$$




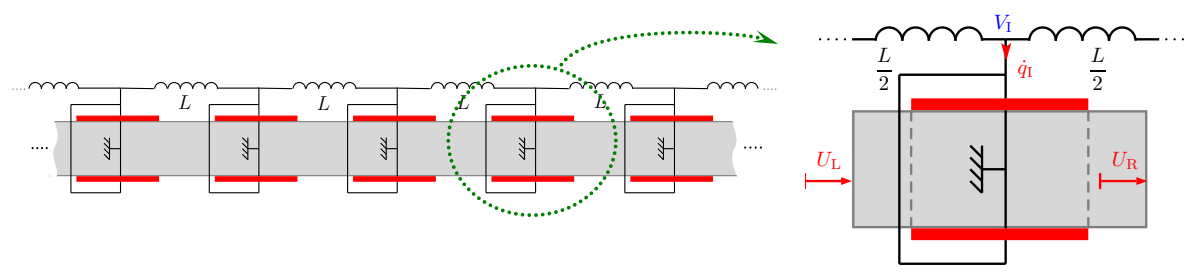

Figure 3: Rod covered with a periodic array of piezoelectric patches that are interconnected through a second order transmission line.

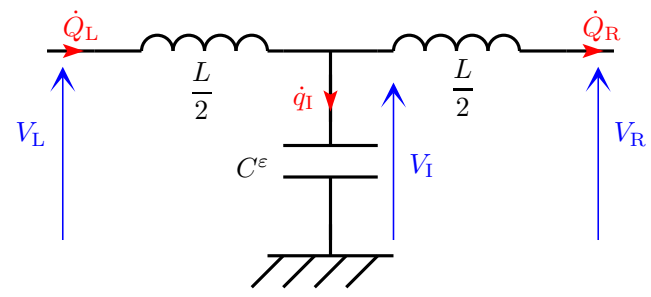

Figure 4: Electrical rod unit cell without piezoelectric coupling.

where $Y$ is the equivalent Young's modulus of the considered element, $\rho$ is the density, $a_{e}$ is its length and $S$ is the cross-sectional area. The corresponding coupling matrix is

$$
\boldsymbol{K}_{\boldsymbol{c}}^{\mathrm{e}}=e\left[\begin{array}{c}
-1 \\
1
\end{array}\right] \text {, }
$$

where $e$ represents the longitudinal piezoelectric coupling coefficient [25, 32. The global matrices $\boldsymbol{K}_{\boldsymbol{m}}, \boldsymbol{M}_{\boldsymbol{m}}$ and $\boldsymbol{K}_{\boldsymbol{c}}$ for the unit cell are then obtained from an assembly of the element matrices with six elements for the segment covered with piezoelectric material plus two purely elastic elements at both ends. This results in a total of 200 elements when considering a succession of 20 unit cells as in the example plotted in Fig. 2. Doubling the number of elements only leads to a $1.3 \%$ decrease of the resonance frequencies around 100 $\mathrm{kHz}$, which justify the use of 10 elements per unit cell.

The displacement and force vectors are

$$
\boldsymbol{q}_{\boldsymbol{m}}=\left[\begin{array}{c}
U_{\mathrm{L}} \\
\boldsymbol{U}_{\mathrm{I}} \\
U_{\mathrm{R}}
\end{array}\right] \quad \text { and } \quad \boldsymbol{F}_{\boldsymbol{m}}=\left[\begin{array}{c}
-N_{\mathrm{L}} \\
\mathbf{0} \\
N_{\mathrm{R}}
\end{array}\right],
$$

where the state variable $U$ is related to longitudinal displacement, $N$ corresponds to the normal force and $\boldsymbol{U}_{\mathrm{I}}=\boldsymbol{q}_{\boldsymbol{m}_{\mathrm{I}}}$ is the mechanical displacement vector of the internal nodes of the unit cell. Considering the electrical part of the unit cell represented in Fig. 4, the line of inductors gives a single degree of freedom on each side of the unit cell, which leads to

$$
\boldsymbol{q}_{\boldsymbol{e}}=\left[\begin{array}{c}
Q_{\mathrm{L}} \\
Q_{\mathrm{R}}
\end{array}\right] \text { and } \boldsymbol{F}_{\boldsymbol{e}}=\left[\begin{array}{c}
V_{\mathrm{L}} \\
-V_{\mathrm{R}}
\end{array}\right],
$$

where $Q$ corresponds to electrical charge displacements and $V$ is used for voltage variables. Note the opposite signs in the electrical and mechanical force vectors that is due to the fact that the voltage $V$ is equivalent to the opposite of the normal force $N$ 25]. From the topology of the unit cell in Fig. 4 and from the inductive and capacitive contributions to the total electrical energy, we obtain

$$
q_{\mathrm{I}}=Q_{\mathrm{L}}-Q_{\mathrm{R}}
$$

and

$$
E_{e}=\frac{\left(Q_{\mathrm{L}}-Q_{\mathrm{R}}\right)^{2}}{2 C^{\varepsilon}}+\frac{L}{4} \dot{Q}_{\mathrm{L}}^{2}+\frac{L}{4} \dot{Q}_{\mathrm{R}}^{2}
$$




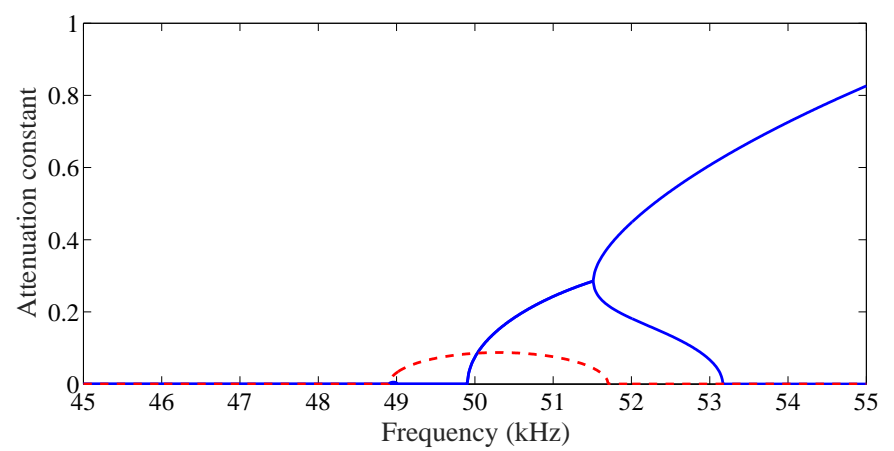

Figure 5: Attenuation constants - (--) without electrical network, $(-)$ with $L=1.2 \mathrm{mH}$.

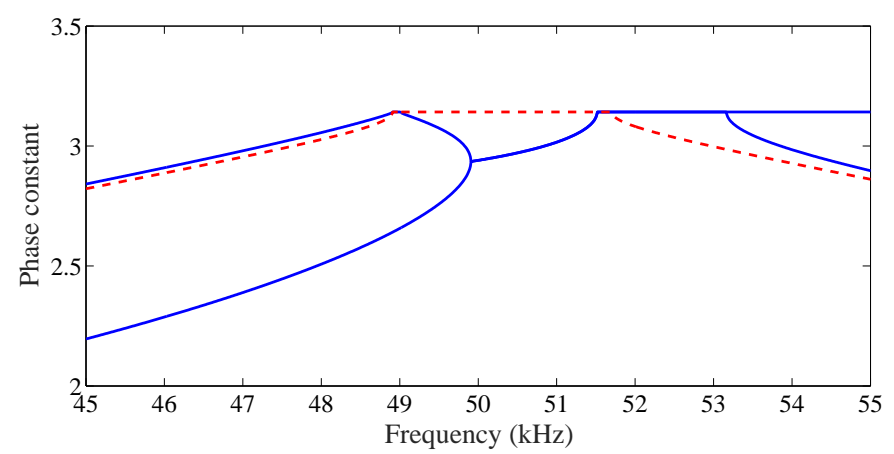

Figure 6: Phase constants - (--) without electrical network, $(-)$ with $L=1.2 \mathrm{mH}$.

Then, Eqs. 111 and (12) give

$$
\boldsymbol{K}_{\boldsymbol{e}}=\frac{1}{C^{\varepsilon}}\left[\begin{array}{cc}
1 & -1 \\
-1 & 1
\end{array}\right], \quad \boldsymbol{M}_{\boldsymbol{e}}=\frac{L}{2}\left[\begin{array}{ll}
1 & 0 \\
0 & 1
\end{array}\right]
$$

and

$$
\boldsymbol{S}=\left[\begin{array}{ll}
1 & -1
\end{array}\right]^{T} .
$$

In the end, the global DSM in Eq. 22 is computed and reorganized into a TM by considering left and right displacement vectors

$$
\boldsymbol{q}_{\mathrm{L}}=\left[\begin{array}{c}
U_{\mathrm{L}} \\
Q_{\mathrm{L}}
\end{array}\right], \boldsymbol{q}_{\mathrm{R}}=\left[\begin{array}{c}
U_{\mathrm{R}} \\
Q_{\mathrm{R}}
\end{array}\right]
$$

and force vectors

$$
\boldsymbol{F}_{\mathrm{L}}=\left[\begin{array}{c}
-N_{\mathrm{L}} \\
V_{\mathrm{L}}
\end{array}\right], \quad \boldsymbol{F}_{\mathrm{R}}=\left[\begin{array}{c}
N_{\mathrm{R}} \\
-V_{\mathrm{R}}
\end{array}\right] \text {. }
$$

This gives a relation between two state vectors including both mechanical and electrical variables:

$$
\left[\begin{array}{c}
U_{\mathrm{R}} \\
Q_{\mathrm{R}} \\
N_{\mathrm{R}} \\
-V_{\mathrm{R}}
\end{array}\right]=\boldsymbol{T}\left[\begin{array}{c}
U_{\mathrm{L}} \\
Q_{\mathrm{L}} \\
N_{\mathrm{L}} \\
-V_{\mathrm{L}}
\end{array}\right] .
$$

Once the TM is obtained, its eigenvalues lead to the attenuation and phase constants. The following numerical calculations are based on the same geometry and material constants as in Refs. 25] and [45] which offer analytical solutions that have been compared to the present numerical results for validation. Twenty 


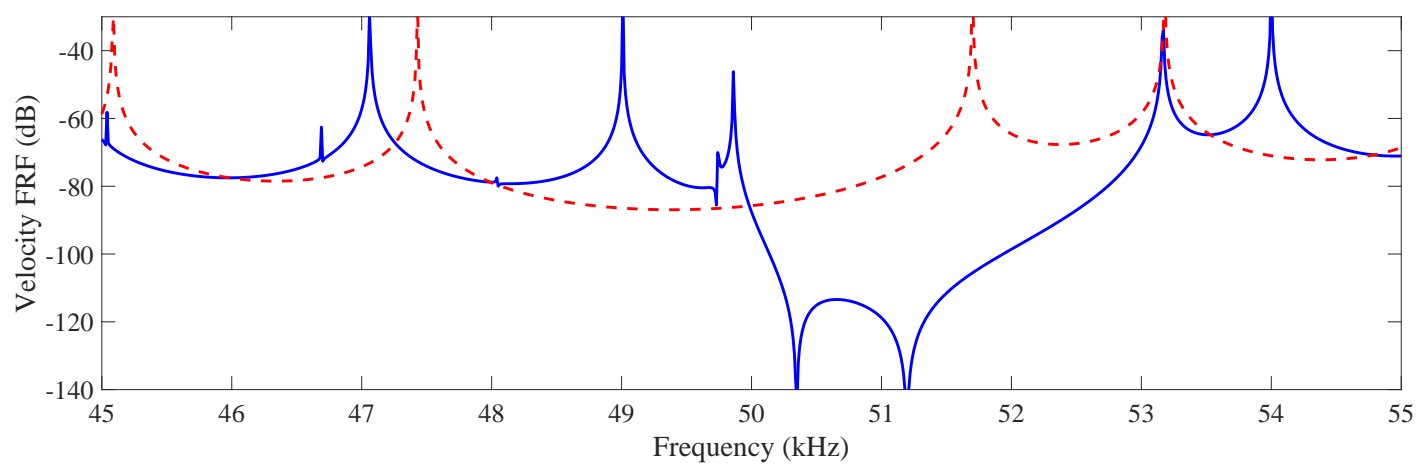

Figure 7: Frequency response functions - (--) without electrical network, $(-)$ with $L=1.2 \mathrm{mH}$.

unit cells similar to that in Fig. 3 form a free-free rod coupled to a line of inductors whose both ends are short circuited. The objective is to show that propagation constants and frequency response functions can be computed for such an electromechanical waveguide. The application of the proposed method combining WFE and RTM methods leads to the results given Figs. 5 , 6 and 7, where the velocity frequency response functions corresponds to the velocity amplitude at one end of the rod when applying a unitary harmonic force at the other end. While the analysis of the results goes beyond the scope of the present paper that focuses on the computational method, it can still be noticed that the coupling to an electrical line strongly modifies propagation constants. For this specific example, the original purely mechanical band-gap [7, 29] is replaced by an electromechanical band-gap corresponding to a larger attenuation constant. The effect on the frequency response function of a free-free rod made of 20 unit cells is illustrated in Fig. 7 where we see a strong reduction of vibration amplitude between 50 and $53 \mathrm{kHz}$. All of this shows that the proposed numerical model can be used for analyzing the coupling between mechanical and electrical band-gaps when considering propagation in electromechanical waveguides [27, 28, 30, 31. At relatively high frequency, one can question the validity of the considered one-dimensional rod model which is based on rigid cross-sections. Yet, the proposed method does not induce any restriction regarding the complexity of the mechanical unit cell and the number of degrees of freedom. The considered stiffness, mass and coupling matrices can actually come from any FE package to enable more refined computation.

\subsection{Beam coupled to a fourth order transmission line}

The second example focuses on a beam that is coupled to an electrical network as represented in Fig. 8 and fully described in Ref. [26]. The electrical network involves transformers of ratio $\hat{a}$ and inductors $L$ in order to build a fourth order transmission line 43 that synthetizes the passive electrical analogue of a beam [46]. When dealing with a beam FE model based on cubic shape functions for the transverse displacement

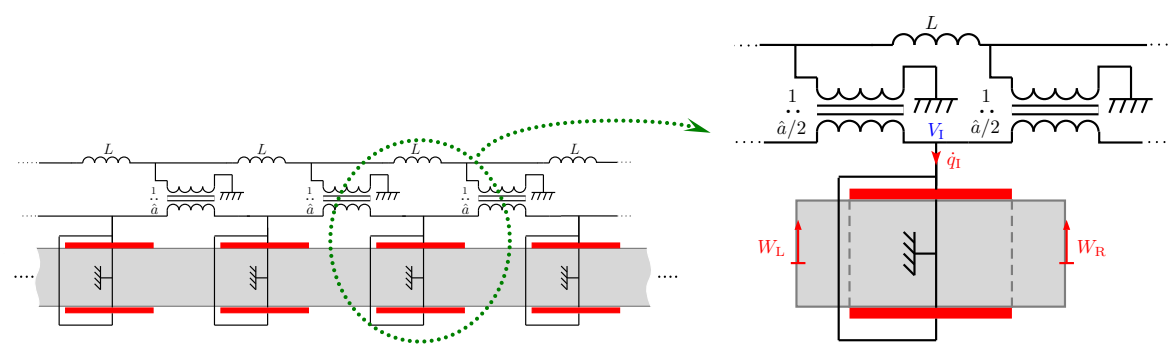

Figure 8: Beam covered with a periodic array of piezoelectric patches that are interconnected through a fourth order transmission line. 


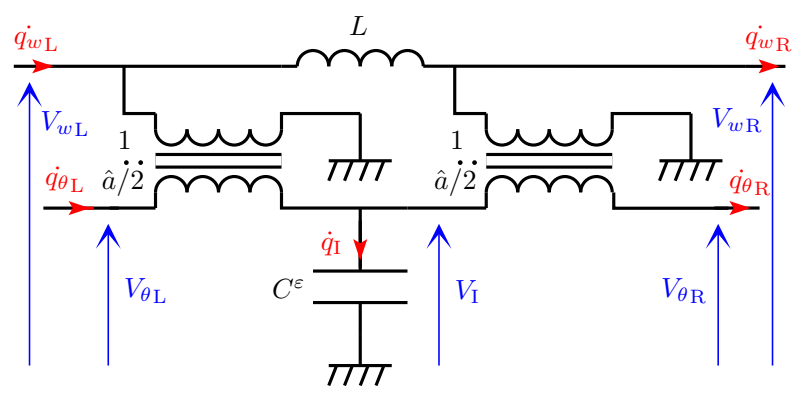

Figure 9: Electrical beam unit cell without piezoelectric coupling.

[24, the element mass matrix is

$$
\boldsymbol{M}_{\boldsymbol{m}}^{\mathrm{e}}=\frac{\rho S a_{e}}{420}\left[\begin{array}{cccc}
156 & 22 a_{e} & 54 & -13 a_{e} \\
22 a_{e} & 4 a_{e}^{2} & 13 a_{e} & -3 a_{e}^{2} \\
54 & 13 a_{e} & 156 & -22 a_{e} \\
-13 a_{e} & -3 a_{e}^{2} & -22 a_{e} & 4 a_{e}^{2}
\end{array}\right]
$$

while the element stiffness matrix is

$$
\boldsymbol{K}_{\boldsymbol{m}}^{\mathrm{e}}=\frac{Y I}{a_{e}^{3}}\left[\begin{array}{cccc}
12 & 6 a_{e} & -12 & 6 a_{e} \\
6 a_{e} & 4 a_{e}^{2} & -6 a_{e} & 2 a_{e}^{2} \\
-12 & -6 a_{e} & 12 & -6 a_{e} \\
6 a_{e} & 2 a_{e}^{2} & -6 a_{e} & 4 a_{e}^{2}
\end{array}\right]
$$

where $I$ is the second moment of area of the beam element of length $a_{e}$. Just as the previous rod example, 10 elements are employed to discretize the beam unit cell. This is fully sufficient because a faster convergence is ensured by the cubic shape functions.

The displacement and force vectors for the whole beam unit cell are represented by

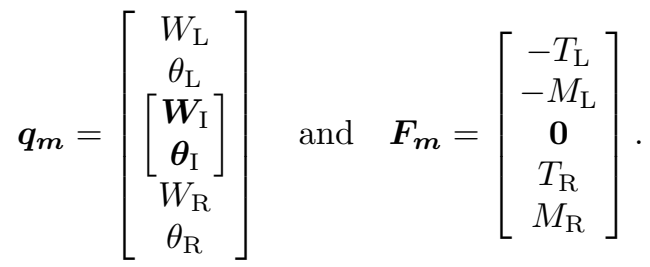

$W$ is the transverse displacement, $\theta$ represents the rotation of the cross-section and $\left[\begin{array}{ll}\boldsymbol{W}_{\mathrm{I}} & \boldsymbol{\theta}_{\mathrm{I}}\end{array}\right]^{T}=\boldsymbol{q}_{\boldsymbol{m}}$ is the displacement vector of the internal nodes of the unit cell. In the force vector, $T$ is the shear force and $M$ is the bending moment. Both are defined as forces applied from the right to the left, which explain the minus signs in the force vector that includes external forcing terms. Then, if the beam element is subjected to piezoelectric coupling, the coupling matrix can be defined as

$$
\boldsymbol{K}_{\boldsymbol{c}}^{\mathrm{e}}=e\left[\begin{array}{llll}
0 & 1 & 0 & -1
\end{array}\right]^{T},
$$

where $e$ is the piezoelectric coupling coefficient related to bending motion 24, 26, 33].

The purely electrical unit cell represented in Fig. 9 leads to electric charge displacement vector and the voltage vectors as

$$
\boldsymbol{q}_{\boldsymbol{e}}=\left[\begin{array}{c}
q_{w_{\mathrm{L}}} \\
q_{\theta \mathrm{L}} \\
q_{w_{\mathrm{R}}} \\
q_{\theta \mathrm{R}}
\end{array}\right] \quad \text { and } \quad \boldsymbol{F}_{\boldsymbol{e}}=\left[\begin{array}{c}
V_{w \mathrm{~L}} \\
V_{\theta \mathrm{L}} \\
-V_{w \mathrm{R}} \\
-V_{\theta \mathrm{R}}
\end{array}\right],
$$




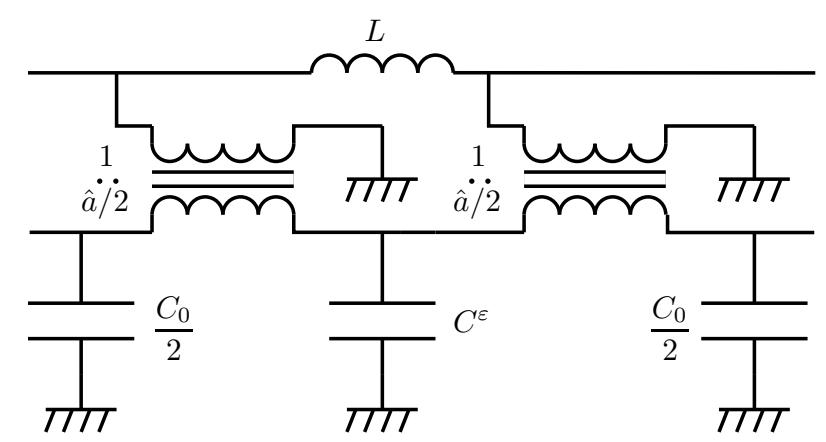

Figure 10: Modified unit cell in the electrical domain.

where the electric charge displacements $q_{w}$ and $q_{\theta}$ are the analogues of the linear of angular mechanical displacements $W$ and $\theta$, while the opposite of the voltage contributions $V_{w}$ and $V_{\theta}$ are the analogues of the force $T$ and moment $M$, respectively [26. Furthermore, the relation between the electric charge displacements at both sides of the unit cell and the charge on the electrodes of the piezoelectric patch $q_{\mathrm{I}}=q_{\theta \mathrm{L}}-q_{\theta \mathrm{R}}$ gives

$$
\boldsymbol{S}=\left[\begin{array}{llll}
0 & 1 & 0 & -1
\end{array}\right]^{T} .
$$

The next step is to define the equivalent of mass and stiffness matrices in the electrical domain. $\boldsymbol{M}_{\boldsymbol{e}}$ can be found from the network in Fig. 9 by defining the magnetic energy $E_{e}^{L}$ as a function of both left and right electric charge displacements. If $\dot{q}_{i}$ is the current flowing through the inductor $L$, the magnetic energy is $E_{e}^{L}=L \dot{q}_{i}{ }^{2} / 2$. We also know from the topology of the network that

$$
\dot{q}_{i}=\dot{q}_{w \mathrm{~L}}+\frac{\hat{a}}{2} \dot{q_{\theta \mathrm{L}}}=\dot{q}_{w \mathrm{R}}-\frac{\hat{a}}{2} \dot{q}_{\theta \mathrm{R}}
$$

which gives

$$
E_{e}^{L}=\frac{L}{4}\left[\left(\dot{q_{w \mathrm{~L}}}+\frac{\hat{a}}{2} \dot{q_{\theta} \mathrm{L}}\right)^{2}+\left(\dot{q_{w \mathrm{R}}}-\frac{\hat{a}}{2} \dot{q_{\theta \mathrm{R}}}\right)^{2}\right] .
$$

In the end, from the expression of the magnetic energy in Eq. 12, we obtain

$$
\boldsymbol{M}_{\boldsymbol{e}}=\frac{L}{2}\left[\begin{array}{cccc}
1 & \hat{a} / 2 & 0 & 0 \\
\hat{a} / 2 & \hat{a}^{2} / 4 & 0 & 0 \\
0 & 0 & 1 & -\hat{a} / 2 \\
0 & 0 & -\hat{a} / 2 & \hat{a}^{2} / 4
\end{array}\right]
$$

On the other hand, the matrix $\boldsymbol{K}_{\boldsymbol{e}}$ cannot be obtained directly from energy consideration when focusing on the unit cell in Fig. 9. As mentionned in Ref. 47] that focuses on a two-dimensional extension of the present one-dimensional network, $\boldsymbol{K}_{\boldsymbol{e}}$ is actually not defined unless we introduce additional degrees of freedom. This can be performed by adding virtual capacitors $C_{0} / 2$ at both ends of the unit cell as shown in Fig. 10 The interest of this modification can be understood by looking at the lattice model in Fig. 11 that is the mechanical analogue of the modified electrical unit cell 26. When $C_{0}$ tends toward zero, it is observed that the displacements and angles at both ends of the unit cell cannot be chosen independently because the kinematics enforces $W_{\mathrm{R}}-W_{\mathrm{L}}=\frac{a}{2}\left(\theta_{\mathrm{L}}+\theta_{\mathrm{R}}\right)$. This constraint is relaxed by adding compliance through the springs $2 / C_{0}$. The stiffness matrix $\boldsymbol{K}_{\boldsymbol{e}}$ of the modified electrical unit cell in Fig. 10 is thus the same as the static stiffness matrix of the mechanical unit cell in Fig. 11 with the additional springs $2 / C_{0}$ placed at the extreme ends of the levers of length $\hat{a} / 2$. From symbolic computation based on the discrete equation 


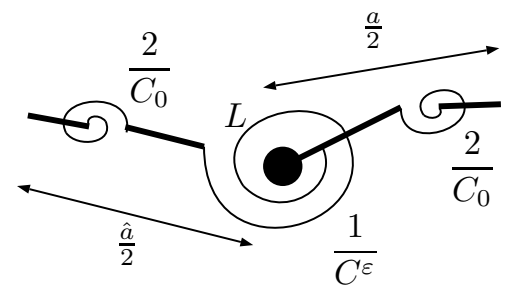

Figure 11: Modified unit cell in the mechanical domain.

describing the modified electrical unit cell or its mechanical analogue, one finds

$$
\boldsymbol{K}_{\boldsymbol{e}}=\frac{4}{\hat{a}^{2} C_{0}}\left[\begin{array}{ccccc}
1 & \frac{\hat{a}}{2} & -1 & \frac{\hat{a}}{2} \\
\frac{\hat{a}}{2} & \frac{\hat{a}^{2}}{4} \frac{C^{\varepsilon}+2 C_{0}}{C^{\varepsilon}+C_{0}} & -\frac{\hat{a}}{2} & \frac{\hat{a}^{2}}{4} \frac{C^{\varepsilon}}{C^{\varepsilon}+C_{0}} \\
-1 & -\frac{\hat{a}}{2} & 1 & -\frac{\hat{a}}{2} \\
\frac{\hat{a}}{2} & \frac{\hat{a}^{2}}{4} \frac{\hat{C}^{\varepsilon}}{C^{\varepsilon}+C_{0}} & -\frac{\hat{a}}{2} & \frac{\hat{a}^{2}}{4} \frac{C^{\varepsilon}+2 C_{0}}{C^{\varepsilon}+C_{0}}
\end{array}\right]
$$

Note that the capacitance $C_{0}$ is a numerical parameter that has to be small compared to $C^{\varepsilon}$. A good practice is to set $C_{0}$ between $C^{\varepsilon} \times 10^{-3}$ and $C^{\varepsilon} \times 10^{-9}$ to conceal its influence on electrical propagation and to avoid numerical issues.

Once the mechanical, electrical and coupling matrices are defined independently, the electromechanical DSM can be computed from Eq. (22) before applying the WFE method. Here the displacement vectors are

$$
\boldsymbol{q}_{\mathrm{L}}=\left[\begin{array}{c}
W_{\mathrm{L}} \\
\theta_{\mathrm{L}} \\
q_{w \mathrm{~L}} \\
q_{\theta_{\mathrm{L}}}
\end{array}\right], \quad \boldsymbol{q}_{\mathrm{R}}=\left[\begin{array}{c}
W_{\mathrm{R}} \\
\theta_{\mathrm{R}} \\
q_{w \mathrm{R}} \\
q_{\theta \mathrm{R}}
\end{array}\right],
$$

and the force vectors are

$$
\boldsymbol{F}_{\mathrm{L}}=\left[\begin{array}{c}
-T_{\mathrm{L}} \\
-M_{\mathrm{L}} \\
V_{w \mathrm{~L}} \\
V_{\theta \mathrm{L}}
\end{array}\right], \quad \boldsymbol{F}_{\mathrm{R}}=\left[\begin{array}{c}
T_{\mathrm{R}} \\
M_{\mathrm{R}} \\
-V_{w \mathrm{R}} \\
-V_{\theta \mathrm{R}}
\end{array}\right]
$$

This finally leads to a TM that gives a relation between electromechanical state vectors as follows,

$$
\left[\begin{array}{c}
W_{\mathrm{R}} \\
\theta_{\mathrm{R}} \\
q_{w \mathrm{R}} \\
q_{\theta \mathrm{R}} \\
T_{\mathrm{R}} \\
M_{\mathrm{R}} \\
-V_{w \mathrm{R}} \\
-V_{\theta \mathrm{R}}
\end{array}\right]=\boldsymbol{T}\left[\begin{array}{c}
W_{\mathrm{L}} \\
\theta_{\mathrm{L}} \\
q_{w \mathrm{~L}} \\
q_{\theta \mathrm{L}} \\
T_{\mathrm{L}} \\
M_{\mathrm{L}} \\
-V_{w \mathrm{~L}} \\
-V_{\theta \mathrm{L}}
\end{array}\right] .
$$

Propagation constants are computed and the RTM method is employed to obtain frequency response functions. While bandgap phenomenons have been highlighted in the previous longitudinal example, it is here chosen to focus on analogous coupling that occurs at lower frequency. Such an analogous coupling can also be investigated for a rod as shown in Ref. [25. In the beam example, the geometry is still the same but bending wave propagation is considered and the velocity frequency response functions corresponds to the 


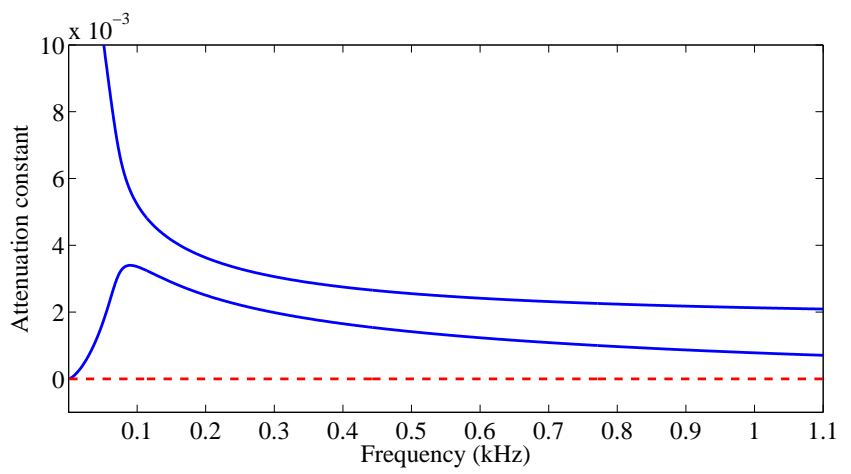

Figure 12: Attenuation constants - (--) without electrical network, $(-)$ with analogous electrical network.

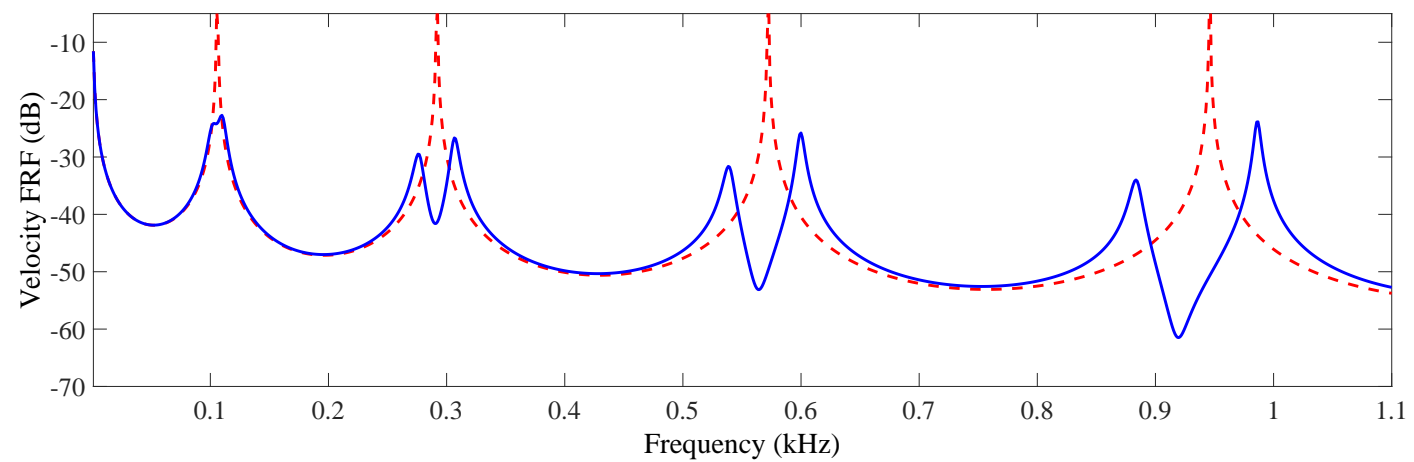

Figure 13: Frequency response function - (--) without electrical network, $(-)$ with analogous electrical network.

free-end transverse velocity $\dot{W}_{n}$ for a unitary harmonic shear force $T_{0}$ at the excited end. Both ends of the electrical line are short-circuited, the transformer ratio $\hat{a}$ is equal to one and the inductance values are all equal to $210 \mathrm{mH}$ in order to ensure a multimodal coupling condition [26]. A resistance $R=20 \Omega$ is also added in series with each inductor to increase damping in the electromechanical structure. While damping is not explicitly introduced in the proposed formulation, impedance calculations show that $L$ simply needs to be replaced by $L-\mathrm{j} R / \omega$ during the computation process.

The two lower attenuation constants are represented in Fig. 12 when considering the beam unit cell coupled to its analogous electrical network. It is remarked that none of the attenuation constant is equal to zero, while it is the case when no coupling occurs. This explains the results related to the dynamics of the whole electromechanical system as shown in Fig. 13 , where we note the broadband damping induced by such analogous piezoelectric coupling. Recalling that the aim of the present work is to propose a computational method and not to deeply interpret the results, interested readers may consider Ref. 45] for more details about multimodal damping with analogous piezoelectric networks. Another remark concerns the fact that a combination of the two cases presented in this section gives access to other configurations as a beam coupled to a second order transmission line or rod coupled to a fourth order transmission line. Investigations on other electrical networks only require the determination of their respective $\boldsymbol{K}_{\boldsymbol{e}}$ and $\boldsymbol{M}_{\boldsymbol{e}}$ matrices. Furthermore, while the proposed examples are based on simple rod and beams models, more complex structures can definitely be considered by extracting their mass, stiffness and coupling matrices from a FE package.

\section{Conclusions}

An electromechanical FE model is proposed for piezoelectrically coupled unit cells involving external electrical degrees of freedom. A charge displacement vector and a voltage vector are defined and related 
to each other through the equivalent of mass and stiffness matrices that are obtained from the topology of the electrical network. Then, it is shown that energy considerations lead to the definition of a DSM that represents the whole electromechanical unit cell. From this novel formulation, the WFE method is directly applied by considering electromechanical state vectors instead of purely mechanical ones. This offers a way to analyze the coupling between mechanical and electrical waveguides from the computation of propagation constants. For frequency response functions, the RTM method turn out to be very efficient for solving numerical instabilities related to the two-point boundary value electromechanical problem. In the end, it is observed that interconnected networks generate a coupling between mechanical and electrical band-gaps, which can be of interest for the control of wave propagation in periodic structures. Moreover, the proposed technique enables the study of electromechanical interactions at lower frequencies such as broadband damping obtained from the coupling of a mechanical structure to its analogous electrical network.

Future work will focus on the use of mass, stiffness and coupling matrices coming from a conventional FE package in order to investigate propagation in one-dimensional periodic waveguides whose unit cell is defined from a three-dimensional model. An extension of the method to two-dimensional periodic structures is also considered for the analysis of low-frequency vibration and wave propagation in electromechanical plates.

\section{References}

[1] M. S. Rao, S. Narayanan, Active control of wave propagation in multi-span beams using distributed piezoelectric actuators and sensors, Smart Materials and Structures 16 (2007) 2577.

[2] L. Ding, H. Zhu, T. Yin, Wave propagation in a periodic elastic-piezoelectric axial-bending coupled beam, Journal of Sound and Vibration 332 (2013) 6377-6388.

[3] O. Thorp, M. Ruzzene, A. Baz, Attenuation and localization of wave propagation in rods with periodic shunted piezoelectric patches, Smart Materials and Structures 10 (2001) 979.

[4] S. Chen, J. Wen, G. Wang, D. Yu, X. Wen, Improved modeling of rods with periodic arrays of shunted piezoelectric patches, Journal of Intelligent Material Systems and Structures 23 (2012) 1613-1621.

[5] O. Thorp, M. Ruzzene, A. Baz, Attenuation of wave propagation in fluid-loaded shells with periodic shunted piezoelectric rings, Smart Materials and Structures 14 (2005) 594.

[6] L. Airoldi, M. Ruzzene, Design of tunable acoustic metamaterials through periodic arrays of resonant shunted piezos, New Journal of Physics 13 (2011) 113010.

[7] L. Airoldi, M. Ruzzene, Wave propagation control in beams through periodic multi-branch shunts, Journal of Intelligent Material Systems and Structures 22 (2011) 1567-1579.

[8] G. Wang, S. Chen, J. Wen, Low-frequency locally resonant band gaps induced by arrays of resonant shunts with Antoniou's circuit: experimental investigation on beams, Smart Materials and Structures 20 (2011) 015026.

[9] W. Zhou, Y. Wu, L. Zuo, Vibration and wave propagation attenuation for metamaterials by periodic piezoelectric arrays with high-order resonant circuit shunts, Smart Materials and Structures 24 (2015) 065021.

[10] S. Chen, G. Wang, Wave propagation in beams with anti-symmetric piezoelectric shunting arrays, Chinese Physics B 25 (2016) 034301.

[11] G. Wang, J. Wang, S. Chen, J. Wen, Vibration attenuations induced by periodic arrays of piezoelectric patches connected by enhanced resonant shunting circuits, Smart Materials and Structures 20 (2011) 125019.

[12] H. Zhang, J. Wen, S. Chen, G. Wang, X. Wen, Flexural wave band-gaps in phononic metamaterial beam with hybrid shunting circuits, Chinese Physics B 24 (2015) 036201.

[13] M. Lallart, L. Yan, C. Richard, D. Guyomar, Damping of periodic bending structures featuring nonlinearly interfaced piezoelectric elements, Journal of Vibration and Control 22 (2016) 3930-3941.

[14] B. Bao, D. Guyomar, M. Lallart, Vibration reduction for smart periodic structures via periodic piezoelectric arrays with nonlinear interleaved-switched electronic networks, Mechanical Systems and Signal Processing 82 (2017) $230-259$.

[15] M. Collet, K. A. Cunefare, M. N. Ichchou, Wave motion optimization in periodically distributed shunted piezocomposite beam structures, Journal of Intelligent Material Systems and Structures 20 (2009) 787-808.

[16] Y. Fan, M. Collet, M. Ichchou, L. Li, O. Bareille, Z. Dimitrijevic, A wave-based design of semi-active piezoelectric composites for broadband vibration control, Smart Materials and Structures 25 (2016) 055032.

[17] D. J. Mead, Wave propagation in continuous periodic structures: research contribution from southampton, 1964-1995, Journal of Sound and Vibration 190 (1996) 495-524.

[18] B. R. Mace, D. Duhamel, M. J. Brennan, L. Hinke, Finite element prediction of wave motion in structural waveguides, The Journal of the Acoustical Society of America 117 (2005) 2835-2843.

[19] D. Duhamel, B. Mace, M. Brennan, Finite element analysis of the vibrations of waveguides and periodic structures, Journal of Sound and Vibration 294 (2006) $205-220$.

[20] J.-M. Mencik, On the low- and mid-frequency forced response of elastic structures using wave finite elements with onedimensional propagation, Computers \& Structures 88 (2010) $674-689$. 
[21] P. B. a. Silva, J.-M. Mencik, J. R. de Franca Arruda, Wave finite element-based superelements for forced response analysis of coupled systems via dynamic substructuring, International Journal for Numerical Methods in Engineering 107 (2016) 453-476. Nme.5176.

[22] T. L. Huang, M. N. Ichchou, O. A. Bareille, M. Collet, M. Ouisse, Traveling wave control in thin-walled structures through shunted piezoelectric patches, Mechanical Systems and Signal Processing 39 (2013) 59-79.

[23] Y. Fan, M. Collet, M. Ichchou, L. Li, O. Bareille, Z. Dimitrijevic, Enhanced wave and finite element method for wave propagation and forced response prediction in periodic piezoelectric structures, Chinese Journal of Aeronautics 30 (2017) $75-87$.

[24] O. Thomas, J.-F. Deü, J. Ducarne, Vibrations of an elastic structure with shunted piezoelectric patches: efficient finite element formulation and electromechanical coupling coefficients, International Journal for Numerical Methods in Engineering 80 (2009) 235-268.

[25] B. Lossouarn, M. Aucejo, J.-F. Deü, Multimodal coupling of periodic lattices and application to rod vibration damping with a piezoelectric network, Smart Materials and Structures 24 (2015) 045018.

[26] B. Lossouarn, J. F. Deü, M. Aucejo, Multimodal vibration damping of a beam with a periodic array of piezoelectric patches connected to a passive electrical network, Smart Materials and Structures 24 (2015) 115037.

[27] A. E. Bergamini, M. Zündel, E. A. Flores Parra, T. Delpero, M. Ruzzene, P. Ermanni, Hybrid dispersive media with controllable wave propagation: A new take on smart materials, Journal of Applied Physics 118 (2015) 154310.

[28] E. A. F. Parra, A. Bergamini, B. V. Damme, P. Ermanni, Controllable wave propagation of hybrid dispersive media with lc high-pass and band-pass networks, Applied Physics Letters 110 (2017) 184103.

[29] Y. Lu, J. Tang, Electromechanical tailoring of structure with periodic piezoelectric circuitry, Journal of Sound and Vibration 331 (2012) 3371-3385.

[30] L. Yan, B. Bao, D. Guyomar, M. Lallart, Periodic structure with interconnected nonlinear electrical networks, Journal of Intelligent Material Systems and Structures (2016) 1045389X16649448.

[31] B. Bao, D. Guyomar, M. Lallart, Piezoelectric metacomposite structure carrying nonlinear multilevel interleavedinterconnected switched electronic networks, Composite Structures 161 (2017) 308 - 329.

[32] J.-F. Deü, B. Lossouarn, M. Aucejo, Comparison of electromechanical transfer matrix models for passive damping involving an array of shunted piezoelectric patches, in: Proceedings of the 22nd International Congress on Sound and Vibration, 2015.

[33] B. Lossouarn, M. Aucejo, J.-F. Deü, Transverse wave propagation in a one-dimensional structure coupled to its electrical analogue: Comparison of transfer matrix models, in: Proceedings of the VII European Congress on Computational Methods in Applied Sciences and Engineering, 2016.

[34] B. Lossouarn, M. Aucejo, J.-F. Deü, Wave finite element method for electromechanical periodic waveguides, in: Proceedings of the 8th ECCOMAS Thematic Conference on Smart Structures and Materials, SMART $2017,2017$.

[35] G. Horner, W. Pilkey, The riccati transfer matrix method, Journal of Mechanical Design 100 (1978) 297-302.

[36] X. Huiyn, A combined dynamic finite element-riccati transfer matrix method for solving non-linear eigenproblems of vibrations, Computers \& Structures 53 (1994) 1257 - 1261.

[37] H. Xue, A combined finite element-riccati transfer matrix method in frequency domain for transient structural response, Computers \& Structures 62 (1997) $215-220$.

[38] N. Stephen, On the riccati transfer matrix method for repetitive structures, Mechanics Research Communications 37 (2010) 663-665.

[39] S. Nanthakumar, T. Lahmer, X. Zhuang, H. S. Park, T. Rabczuk, Topology optimization of piezoelectric nanostructures, Journal of the Mechanics and Physics of Solids 94 (2016) 316-335.

[40] S. Nanthakumar, T. Lahmer, X. Zhuang, G. Zi, T. Rabczuk, Detection of material interfaces using a regularized level set method in piezoelectric structures, Inverse Problems in Science and Engineering 24 (2016) $153-176$.

[41] A. Bloch, Electromechanical analogies and their use for the analysis of mechanical and electromechanical systems, Journal of the Institution of Electrical Engineers - Part I: General 92 (1945) 157-169.

[42] W. X. Zhong, F. W. Williams, On the direct solution of wave propagation for repetitive structures, Journal of Sound and Vibration 181 (1995) 485-501.

[43] C. Maurini, F. dell'Isola, D. Del Vescovo, Comparison of piezoelectronic networks acting as distributed vibration absorbers, Mechanical Systems and Signal Processing 18 (2004) 124-1271.

[44] L. Brillouin, Wave propagation in periodic structures, McGraw-Hill, 1946.

[45] B. Lossouarn, Multimodal vibration damping of structures coupled to their analogous piezoelectric networks, Theses, Conservatoire national des arts et metiers - CNAM, 2016. URL: https://tel.archives-ouvertes.fr/tel-01507059

[46] M. Porfiri, F. dell'Isola, F. M. Frattale Mascioli, Circuit analog of a beam and its application to multimodal vibration damping, using piezoelectric transducers, International Journal of Circuit Theory and Applications 32 (2004) $167-198$.

[47] B. Lossouarn, M. Aucejo, J.-F. De, K. A. Cunefare, Design of a passive electrical analogue for piezoelectric damping of a plate, Journal of Intelligent Material Systems and Structures 0 (In Press) 1045389X17731232. 